\title{
TRANSPORTE Y COMERCIO: EL ARBITRAJE EN LONDRES. EN LA ACTUALIDAD
}

Ed Patton y Jonathan Pitman*

El volumen de arbitraje comercial que se efectúa en Londres es muchísimo mayor que el que se realiza en cualquier otra ciudad del mundo. En parte, las razones que para ello existen son históricas, teniendo en cuenta principalmente el establecimiento durante el siglo XIX de muchas florecientes asociaciones de comercialización de mercancías o "commodities" y el desarrollo de Londres como principal centro de seguros y reaseguros comerciales en los sectores de casco, de carga y de $\mathrm{P} \& \mathrm{I}$. Pero el considerable incremento de volumen de arbitraje que se dispensa en esta ciudad desde hace más de treinta años, no se puede adscribir por entero a razones históricas. Ha tenido lugar un gran despliegue de elevadisima pericia por parte de árbitros y abogados, que va unido a la fácil disponibilidad de experiencia técnica de primera clase en cuanto a inspectores, constructores navales, agrónomos, corredores de seguros, corredores de fletes y otros semejantes. Estos factores hacen posible que Londres proporcione un servicio de arbitraje comercial que otros centros encuentran muy difícil de emular.

La legislación de arbitraje en Inglaterra está reunida en las Leyes de Arbitraje de 1950 a 1979, así como en el derecho común. El estatuto principal es el de la Ley de Arbitraje de 1950, que ha sido enmendada por legislación subsiguiente, especialmente por las Leyes de Arbitraje de 1975 y 1979. Pero estas Leyes no suponen un Código completo. Suponen, más bien, una estructura que da a las Cortes poderes de a segurar el cumplimiento de las decisiones de arbitraje y de supervisar el proceso del arbitraje.

* Texto de la Conferencia dictada en la Asociación Peruana de Derecho Marítimo, San Isidro, setiembre de 1987. 
Los principios de derecho común son también de importancia. Por ejemplo, el principio de que un árbitro deba actuar equitativamente y dar a ambas partes la debida oportunidad de presentar sus casos tiene su origen en este derecho común. Pero las Leyes de Arbitraje contienen medios de corrección de que se puede disponer cuando el tribunal arbitral actúa indebidamente. El principio básico - que pertenece al derecho común- es quizá el de que las partes tienen libertad para determinar la jurisdicción del tribunal de arbitraje y el procedimiento que se haya de seguir.

Excepto cuando se indique de otra manera, los comentarios que hacemos en esta disertación son de aplicación general al arbitraje comercial. Pero en esta fase inicial deseamos mencionar tres tipos de arbitraje en que pueden aplicarse reglas especiales debido a la clase de elección que hagan las partes.

1. Juicios de arbitraje de las asociaciones de comercialización de mercancias o "commodities".

Existen numerosas asociaciones dedicadas a la comercialización de mercancías, que tienen su base en Londres. Pueden mencionarse la Asociación Comercial de Grano y Alimentación (GAFTA), la Asociación Federada de Aceites, Semillas y Grasas (FOSFA), la Federación Comercial del Café (CTF) y la Asociación de Azúcar Refinada (RSA). Esta clase de asociaciones publican formas estandarizadas de contratos, que se emplean mucho en el comercio internacional. Por lo general, estos contratos disponen que el arbitraje se celebre en Londres, dentro del reglamento de la asociación correspondiente. Hablando en general, el procedimiento de arbitraje que se estipula en tales reglamentos es poco usual en cuanto se procede en dos etapas. El arbitraje suele tener lugar ante dos miembros de la asociación que (según sean las reglas) pueden ser nombrados por las partes o por la asociación. La fase inicial del arbitraje se desenvuelve de forma muy rápida y sin formalidades, sobre la base única de los documentos presentados y $\sin$ que las partes estén representadas por abogados. No obstante, la parte que no quede satisfecha podrá apelar a una Junta de Apelación, cuyos integrantes se toman de un elenco de personas eminentes en el comercio. En la etapa de apelación, las partes suelen estar representadas por abogados, pero no siempre. Los reglamentos 
de estas asociaciones imponen con frecuencia límites de tiempo muy cortos para comenzar el arbitraje e iniciar la apelación.

2. Aunque el comercio en la mayoría de los géneros de mercancías se regula por diferentes asociaciones, existen algunos sectores comerciales que no se han desarrollado por este camino. En especial se debe hacer referencia al petróleo. Desde principios del decenio 1970 , el comercio del petróleo, tanto crudo como sus productos, como los señores sepan muy bien se ha convertido en un gran negocio internacional. Muchos de los contratos se gobiernan expresamente por la legislación inglesa y están sujetos a arbitraje en Londres. Ocasionalmente, la cláusula de arbitraje dispone que el Reglamento de Arbitraje y Conciliación de la Cámara Internacional de Comercio (CIC) sea el que haya de aplicarse, pero es mucho más corriente que tales arbitrajes se celebren bajo la ley general de arbitraje de Inglaterra. Tales litigios los dirimen a menudo árbitros especialistas en asuntos marítimos, a quien se hace referencia más adelante, o bien por abogados de sala ("barristers") en caso de que haya puntos jurídicos difíciles en el asunto de que se trate.

\section{Arbitraje de salvamento}

La única modalidad normalizada para contratos de salvamento que se emplea en el ámbito internacional es el "Lloyd's Open Form" (cuya versión actual se conoce como LOF 1980). Se trata de un contrato con arreglo al cual los intereses que se benefician del salvamento admiten su responsabilidad al efecto, sujeto a cualquier cuestión que surja acerca del buen exito de los servicios, y se someten a arbitraje respecto a la cuantía. Estos arbitrajes los administra el Departamento de Arbitraje de Salvamento de Lloyd's y siempre se celebran en Londres (Al igual que sucede con los arbitrajes de las Commodities Trade Associations, el procedimiento de arbitraje de salvamento está conducido en dos etapas. El primer arbitraje tiene lugar ante un solo árbitro, que es un Barrister nombrado por Lloyd's en nombre de todas las partes y se escoge de un pequeño elenco de abogados expertos en estas cuestiones. Es poco frecuente que los testigos den testimonio oral. En su lugar, estos arbitrajes se suelen realizar sobre la base de documentos a los que apoyan los argumentos orales de los abogados. Las partes que no queden satisfechas pueden apelar al Arbitro de Apelación, que es 
también un Barrister en activo. En cualquier momento dado, existe un Arbitro de Apelación a quienes se refieren todas las apelaciones de arbitraje en salvamento).

\section{A sociación de Arbitros Maritimos de Londres}

Como reacción al rápido aumento de ia demanda de arbitraje marítimo en Londres, bien se trate de contratos de fletamento, conocimientos de embarque, contratos de remolque, contratos de construcción naval, de compra y venta y otros tipos de contratos maritimos, se estableció en 1960 la Asociación Londinense de Arbitros Marítimos "La Asociación”. Su objeto principal es el de mantener el mejor comportamiento posible en cuanto a ética, práctica y experiencia, dentro de la conducta del arbitraje marítimo. Existen actualmente en la Asociación 45 in tegrantes de pleno derecho, y más de 350 que los secundan en el Reino Unido y el extranjero. Prácticamente todos los árbitros cuentan con una larga experiencia en uno u otro aspecto de las transacciones marítimas y existe cierto número de ellos que ejercen el arbitraje como ocupación exclusiva.

En 1984, La Asociación publicó un reglamento experimental como medida previa a formular un código definitivo proyectado para mejorar al máximo los procesos de arbitraje marítimo. Tras un prolongado proceso de consultación entre los árbitros y los beneficiarios de su labor, intereses comerciales, aseguradores y abogados, se han promulgado y entrado en vigor las Condiciones de 1987. Se trata de un código cuidadosamente pensado que proporciona a los árbitros ciertos poderes de procedimiento para facilitar el funcionamiento del proceso de arbitraje. Las Condiciones de 1987 son aplicables a todos los casos en que los árbitros sean socios de La Asociación y pueden también emplearse en otros casos si así se desea. Volveremos a referirnos a ellos más adelante.

\section{Composición del Tribunal de Arbitraje}

Un contrato o cláusula de arbitraje en que se estipule "arbitraje en Londres" y nada más será perfectamente válido y su efecto es que el arbitraje estará a cargo de un solo árbitro. Desde luego, a menos que el contrato de arbitraje disponga que haya más de un árbitro, su sentido siempre se toma como referencia a un solo árbi- 
tro (1). Sin embargo, lo más frecuente es que las cláusulas arbitrales dispongan de dos árbitros y un eventual tercer árbitro dirimente (normalmente nombrado por los dos arbitros iniciales), o alternativamente un tribunal de tres árbitros. Cuando hay tres árbitros, la decisión se toma por mayoría. Cuando el tercer árbitro es dirimente, la decisión se toma por los dos árbitros iniciales, si están de acuerdo, o de no llegar a este acuerdo la decisiọn la tomará el dirimente. En el arbitraje inglés siempre se ha acostumbrado emplear dos árbitros y un dirimente. Sépase que si el arbitraje toma la forma de un juicio oral, el dirimente será generalmente el que presida la sesión, aunque técnicamente no posee cometido alguno hasta que los dos árbitros disientan entre si.

No es posible que el arbitraje esté demorado por falta de nombramiento del árbitro o árbitros. En caso de haber un árbitro único, y que las partes no puedan convenir en la elección de la persona adecuada o una de las partes rehusase discutir la cuestión o no lo hiciese por otro motivo, el Tribunal Comercial hará los nombramientos necesarios en nombre de las partes, bastando para ello con que lo desee una de las dos (2). Si la decisión se deja a dos árbitros, y cada parte nombra a uno de cllos, el demandante puede notificar al demandado su exigencia de nombrar un árbitro dentro de un plazo de tiempo especificado -que a veces puede ser tan breve como 7 días-y si el demandado no lo hiciese asi, el demandante tendrá derecho a que su árbitro sea el único árbitro (3). En todos los demás casos, la Corte tiene la potestad de hacer un nombramiento siempre que hubiere falta de designación de alguno de los árbitros o el dirimidor (4). Exactamente el mismo procedimiento se sigue cuando no se ha nombrado un substituto en caso de que el árbitro inicial muriese o rehusase actuar, o quedase incapacitado para hacerlo.

(1) Ley de 1950, Art. $6^{\circ}$

(2) Ley de 1950, Art. $10^{\circ}$ (1) (a)

(3) Ley de 1950, Art. $7^{\circ}$

(4) Véase la Ley de 1950, Art. $10^{\circ}$ (enmendada por la Ley de Administración de la Justicia de 1985 , Art. $58^{\circ}$ ). 
En Londres la tendencia que se sigue es la de que los tribunales se compongan de dos árbitros y un dirimente; no obstante, se están usando cada vez más árbitros únicos, pero existe una reticencia de muchos litigantes comerciales a convenir en el nombramiento de un solo árbitro y a veces los abogados no se atreven a aconsejar a sus clientes que se pongan de acuerdo para tal nombramiento.

A nuestro modo de ver, la mayoría de los casos se pueden resolver satisfactoriamente por obra de un solo árbitro, siempre que la persona elegida tenga suficiente experiencia y sea del calibre adecuado. No hay duda de que el empleo de un solo árbitro resulta casi siempre en un arbitraje más rápido, lo que siempre produce un ahorro. De lo contrario debe utilizarse un tribunal de 3 árbitros, o de dos árbitros y un eventual tercer árbitro dirimente en caso de desacuerdo.

En lo que se refiere a retrasos, una característica especial a observar en las Condiciones LMAA (1987) es la de que un árbitro que no pueda ofrecer una fecha razonablemente próxima para efectuar su arbitraje deberá dimitir si se le exige por alguna de las partes litigantes, de forma que se pueda nombrar un substituto.

Los litigantes comerciales creen a menudo que van a estar en desventaja a menos que tengan su "propio" árbitro en el tribunal. En algunos casos, esto puede ser verdad, pero no es frecuente. La ley exige de los árbitros que actúen con imparcialidad y así lo suelen hacer. Otro factor que dichas partes no tienen a menudo en cuenta es el de que, con un tribunal compuesto de dos árbitros y un dirimente, éste será el que tome la decisión definitiva. Igualmente con.un tribunal de tres árbitros, será el tercero el que muy a menudo tome la decisión. Ninguna de las partes tiene control alguno sobre la elección del dirimente o tercer árbitro, aunque ambas hagan la elección de un árbitro único (excepto en los raros casos en que las partes no pueden llegar a un acuerdo, dejando que la clección la haga a la Corte Comercial).

Procedimientos Generales de Arbitraje

Algunos juicios de arbitraje se realizan con pocas formalidades y las partes se limitan a hacer declaraciones escritas a las que apoyan los necesarios documentos, dejando después el tribunal pa- 
ra que tome su decisión (lo que se conoce como un "Award") No es necesario que un caso de arbitraje sea presentado por abogados. No obstante, a menos que el litigio sólo conste de un simple hecho sin que lo afecten aspectos legales de importancia, lo juicioso es que las partes tomen, por lo menos, asesoramiento jurídico y, en casos más complicados, estar legalmente representadas también. Aún cuando haya abogados en el caso, este puede seguir su curso con pocas formalidades si así lo desean las partes. Pero, a menudo. si hay abogados, el procedimiento tiende a asemejarse al seguido en la Corte Comercial, con informes escritos y "descubrimiento de doeumentos" o Discovery, lo que culmina en un juicio oral en el que el argumento jurídico es presentado a los árbitros, con el frecuente complemento de la deposición oral de testigos, $\mathrm{y}$, cuando corresponde, pruebas periciales. No hay duda de que tal procedimiento resulta en que los hechos y puntos de controversia se tratan en gran extensión, elevando al máximo la probabilidad de que el veredicto sea correcto, dentro de lo que la ley estipula. Esto está desde luego justificado cuando la disputa es acerca de grandes sumas de dinero. Sin embargo, si el dinero no es excesivo, los intereses de las partes, en términos de tiempo y gastos, harían aconsejable un proceso menos exhaustivo. Los abogados deberán reconocer la virtud de la flexibilidad en tales casos. Así deben hacerlo también sus clientes.

Esta necesaria flexibilidad está reconocida en las Reglas de 1987. [Por ejemplo, contienen un procedimiento "aconsejable" para gobernar los casos cuya solución se ha de basar en documentos. Y, en relación a los casos que han de someterse a un proceso oral, el reglamento impone a las partes ( $y$, claro está, a sus abogados) el deber de que acuerden un horario a observar en el proceso, dando los árbitros las órdenes adecuadas, haciéndolo así en los casos en que corresponda después de discutirlo en reunión conjunta entre los árbitros y los abogados, pero sólo si las partes no pueden llegar a un acuerdo. Los árbitros pueden también disponer que declaraciones por escrito de los testigos se intercambien entre las partes antes de que comience el juicio oral para que el interrogatorio sea más breve y limitar al mínimo la posibilidad de que haya sorpresas de última hora; asimismo pueden disponer que los expertos en quienes las partes deseen apoyarse como prueba, deberán reunirse para tratar de acotar el terreno de la disputa tienical. 
Para aquellas personas cuya experiencia reside en sistemas de derecho civil, puede ser dif ícil comprender la actitud que adoptan sistemas tales como el derecho común inglés en cuanto a "descubrimiento de documentos". Esto es verdad hasta cierto punto con los abogados, pero mucho más con relación a los clientes. Dentro del procedimiento de las Cortes inglesas, cada una de las partes.debe revelar a la otra todos los documentos que estén en su poder o control y que tengan cualquier clase de conexión con puntos del litigio (5), a menos que el documento goce del privilegio de no exhibirse, por ejemplo, cuando se trata de privilegios profesionales. En relación a los juicios de arbitraje, no existe la misma regla, pero los árbitros tienen poderes discretionarios semejantes (6). Si una de las partes solicitara que se diese una orden de "descubrimiento" esta orden, en general, se dará. Las partes que acudan a Londres para someterse a arbitraje podrán esperar, por lo menos cuando intervengan abogados, que se les pueda exigir el hacer tal descubrimiento.

No se pueden prsentar solamente los documentos que favorecen a la causa propia. Recíprocamente, la parte opuesta no podrá ocultar documentos que prefiera no revelar. En el referido sentido la facultad de descubrimiento es un instrumento muy valioso hacia la consecución de la verdad.

\section{Decisiones en el ínterim}

Hay ocasiones en que resulta ventajoso e incluso necesario en interés de la justicia que se tome rápidamente una decisión pero no necesariamente acerca de todos los puntos en litigio. Cuando así sucede, una decisión en el ínterim podrá ser deseable. Los árbitros tienen potestad para tomar decisiones en el ínterim (7) y, desde luego, en el sector marítimo no tienen inconveniente alguno en hacerlo. Considérense el(los) siguiente(s) ejemplo(s):

(5) Orden 24, Reglamento del Tribunal Supremo.

(6) Ley de 1950, Art. $12^{\circ}$ (1)

(7) Ley de 1950, Art. $14^{\circ}$ 
a) Un buque en fletamento de viaje redondo queda retrasado durante un plazo de tiempo considerable en su puerto de carga y se produce una sobrestadía muy prolongada, la situación hace pensar que el retraso va a continuar indefinidamente. ¿Tendrá que esperar el armador a ef ectuar su demanda hasta después de que el buque se haya cargado, transportado el cargamento y descargado antes de que se le conceda compensación por la sobrestadia? (llamado demorage). Esto no sería justo. En tal situación, los árbitros londinenses estarían dispuestos a escuchar una solicitud para producir una decisión en el interim, que cubra la sobrestadía abonable ya producida, aunque el buque permanezca aún en el puerto de carga, pero siempre y cuando que las disposiciones de sobrestadía del contrato de fletamento no contengan disposiciones que impidan a los árbitros seguir este procedimiento.

b) Un contrato de fletamento para dos viajes consecutivos excluye el transporte de ciertas clases de cargamento,comprendidos los peligrosos; los fletadores designan un cargamento de salitre para el segundo viaje; los armadores desean que se llegue a una decisión arbitral mientras el buque está todavia haciendo su primer viaje para saber si tienen la obligación de cargar el salitre, es decir, desean una decisión declaratoria en el ínterim. Los árbitros de Londres siempre estarán dispuestos a atender tal solicitud (8).

Hay circunstancias en que la urgencia no es un factor de importancia pero en que, no obstante, una decisión en el ínterim puede ser de utilidad. Por ejemplo, cuando esté en disputa un solo punto de la ley acerca de responsabilidades, pero los daños puedan comprender complicados elementos de cuantificación. En tales circunstancias, una decisión tomada aisladamente sobre la cuestión de responsabilidad podría muy bien facilitar una resolución. Puede haber otro caso en que haya poco o nada que disputar acerca de desperfectos, donde, en principio, las respuestas a una o más preguntas podrían simplificar la detallada preparación de la demanda y de la defensa. En ambos ejemplos, los árbitros tendrían potestad para tomar decisiones en el ínterim de la misma forma que la tie-

(8) Basado en National Rumour Compañia S.A. v. Lloyd's-Libra Navegaceo S.A. (1982) 1 Ll. Rep. 472. 
nen en el caso es que una de las partes hace la solicitud. En todos los casos de esta clase, los árbitros poseen la discreción de conceder o rehusar lo solicitado.

\section{Costos}

Los costos de un juicio de arbitraje son de dos clases: en primer lugar, los emolumentos y gastos de los árbitros y en segundo lugar los gastos incurridos por las partes, tales como honorarios legales y honorarios y gastos de los expertos y testigos. Siempre que se realiza un arbitraje, lo normal es que la parte perdedora reciba la orden de pagar las costas de la primera de las categorías, y Londres no es excepción a esta regla. Pero lo que distingue a los juicios de arbitraje ingleses como excepcional es la exigencia, como regla general, de que la parte perdedora abone también los costos de la ganadora. La mayoría de los sistemas o bien no permiten la recuperación de tales costos o sólo permiten una recuperación nominal, con sólo una recuperación importante en circunstancias excepcionales, por ejemplo cuando la parte perdedora ha obrado de mala fe o si su caso ha puesto en evidencia una actitud poco seria. En Inglaterra, la ley prescribe que la parte perdedora abone las costas de la ganadora, a menos que haya razones excepcionales que justifiquen una solución diferente. Por ejemplo donde haya diferentes puntos contenciosos y la parte ganadora hubiese perdido uno o más de ellos, o bien cuando la forma en que se ha conducido el juicio de arbitraje haya elevado innecesariamente los gastos. En tales situaciones, los árbitros podrán privar a la parte ganadora de parte de sus gastos o incluso ordenarla que pague una proporción de los costos de la perdedora (9).

Sería erróneo pensar que la parte ganadora recibe normalmente el $100 \%$ de las costas. Se le concederá el $100 \%$ de todos los emolumentos que razonablemente se paguen a los expertos y otros gastos parecidos. Pero, por lo general, sólo se le concederá cierta proporción de las costas legales. En la práctica, esta proporción se cleva a un 60 ó 70 por ciento.

En la Corte es posible que el demandado deposite un pago

(9) La potestad de los árbitros para adjudicar costas está contenida en la Ley de 1950 , Art. $18^{\circ}$. 
con la Corte para ahorrar costos. Entonces, el demandante tendrá la opción de tomar el pago como ajuste total y definitivo de su reclamación o continuar con el proceso. Si continúa y obtiene una decisión favorable de indemnización no mayor que la cantidad depositada con la Corte por la otra parte, no tendrá derecho a recuperar del demandado ningunas de las costas que se produjesen desde el momento en que hubiese debido aceptar el pago. Adicionalmente tendrá que pagar las costas que el demandante haya abonado después de dicho momento. El mismo objetivo se puede conseguir por un demandado en arbitrajé empleando el recurso conocido como "oferta abierta" o "oferta precintada" ("Open offer" y "sealed offer", respectivamente). Puede parecer extraño que este recurso se conozca con diferentes nombres que parecen tener significados diferentes. La palabra "open" (abierto) indica que la of erta se hace de forma que conste abiertamente, al revés de lo que sucede con una oferta "without prejudice" (sin perjuicio), que se considera como cuestión totalmente privada entre las partes y no puede ponerse en conocimiento de los árbitros. La oferta está precintada ("sealed") en el sentido de que se entrega un ejemplar a los árbitros al final de la vista, con las instrucciones de que no se puede abrir hasta que hayan dado su veredicto, pero antes de que hayan decidido adjudicar las costas. Entonces, incluso si la decisión fuese a favor del demandante, las costas se decidirán a favor del demandado contando a partir del momento en que se hubiese aceptado la oferta en caso de que la decisión estipulase una cantidad no mayor de la de la oferta.

Hay distintas decisiones del tribunal que han aprobado el empleo de este procedimiento (10) su efecto en un arbitraje puedè ser bastante espectacular, puesto que significa, inter alia, que todos los honorarios de los árbitros en relación con la vista son abonables por el demandante. Asi pues, puede actuar como potente instrumento a favor de un acuerdo. Se emplea con mucha frecuencia, aunque no siempre es apropiado. Si la disputa gira en torno a cuestiones de responsabilidad legal siendo predecible el importe de la indemnización, una oferta precintada tiene poco valor. Sin em-

(10) Por ejemplo en el caso del Tramountana Armadora S.A. v. Atlantic Shipping Co. S.A. (1978) 1 L. Rep. 391, y el "IOS I" (1987) 1 L. Rep. 321 . 
bargo, no ocurre así, cuando lo que se disputa es la cuantificación de la indemnización.

\section{Seguridad acerca de costos}

La ley inglesa adopta la posición de que no es justo forzar a un demandado a que se defienda de una reclamación cuando al mismo tiempo, cstá en peligro de perder el importe de las costas si la reclamación no fuese válida. Por este motivo, suele ser posible para el demandado el obtener cierta seguridad acerca de las costas, lo que, en general, tomará la forma de una garantía bancaria o de una Asociación de Defensa o Club de P \& I, donde el demandante proviene del extranjero y no cuenta con cierto volumen de biencs en Inglaterra. El demandante no tiene un derecho semejante a solicitar que se aseguren sus costos en la consecución de su reclamación. No obstante, puede solicitar una seguridad respecto a las costas de defender una contrarreclamación, cuando la introducción de ésta por parte del demandado suscite otros puntos de contención que añadir a los costos del arbitraje. La sanción por no cumplir una orden de aportar garant ías es que la reclamación (o contrarreclamación, según sea el caso) no puede seguir adelante hasta que se ofrezca una garantía.

Las solicitudes de garantía para las costas se hacen normalmente a la Corte, en vez de a los árbitros (11). Los árbitros no tienen potestad para conceder seguridades, a menos que el acuerdo de arbitraje se la confiera. Esto es poco frecuente. Sin embarqo, obsérvese que con arreglo a las Condiciones LMAA (1987) los árbitros cuentan con dicha potestad.

\section{Base de cobrar honorarios}

En Inglaterra no se sigue la práctica de que los abogados o árbitros cobren sus emolumentos sobre la base de un porcentaje. Hay excepciones, en el caso de los árbitros, cuando las partes han acordado someterse a arbitraje, dentro de un reglamento que rija éste, tal como el CIC cuyas reglas imponen una base de porcentalje. Desde luego, según las Reglas del CIC también hay que pagar

(11) Con arreglo a la Iey de 1950 , Art. $12^{\circ}$ (6)

13.4 
un cierto porcentaje administrativo. La mayoria de los abogados comerciales ingleses que están experimentados en cuestiones de arbitraje, consideran que los procesos bajo el Reglamento CIC. constituyen una manera muy cara y poco ágil de resolver los litigios. Aparte de los casos excepcionales, los abogados y árbitros ingleses suclen basar sus honorarios en el tiempo invertido en el asunto y en la complejidad de éste. Quizá se deba decir de paso que los abogados ingleses no siguen la práctica de actuar sobre la base de pagos de contingencia, o tuota litis.

Otro punto que merece observarse es que según las Condiciones LMAA (1987), las partes han de abonar un precio de reserva antes de celebrarse la vista de su causa. En la actualidad, es una cantidad de L 150 por día para cada árbitro. La vista no tendrá lugar si no se ha pagado esta suma. Y si las partes suspenden la vista menos de tres meses antes de la fecha programada para el comienzo. los emolumentos no son recuperables; cuando se dé una notificación de suspensión con adelanto superior a tres meses, se puede obtener la devolución del 50\%.

\section{Intereses}

Los árbitros tienen la potestad de adjudicar intereses sobre la cantidad principal que conceden dentro de una reclamación o contrarreclamación (12). Estos intereses siempre se decidirán a menos que circunstancias excepcionales lo dispongan de otra manera. En general. un demandante puede esperar que le sean abonables intereses deste el momento en que la deuda se originase o en que se produjese la causa del proceso, y hasta la fecha de la decisión arbitral. La ley no estipula que sólo se concedan intereses desde el momento en que comience el juicio de arbitraje o desde la fecha en que se haya hecho una petición formal de alojar una demanda. Del mismo modo, no existe una tarifa prescrita o máxima que restrinja la decisión de los árbitros. La tarifa es a su discreción y. por lo general, decidirán una tarifa comercial razonable.

Desde la fecha de la decisión y hasta que se efectúe el pago, se cuentan intereses sobre el total combinado del principal e int:-

(12) Este es un poder implícito que se basa en la analogía con la potestad de la Corte, en especial con arreglo a la Ley de la Reforma Legal (Disposiciones Varias) 1934 , Art. $3{ }^{\circ}$. 
reses que comprenda la decisión según la misma tarifa que en un juicio ante las Cortes (13) que, en estos momentos es del 15\% anual.

Una potestad que recientemente han adquirido los árbitros es la de conceder intereses sobre cualquier cantidad reclamada durante el proceso, aunque la suma principal se haya pagado en el momento en que haya comenzado el arbitraje (14). Hace ya mucho tiempo que muchos árbitros y otras personas interesadas han pensado que la carencia de tal potestad suponía un defecto importante dentro del sistema de arbitraje. Es evidentemente correcto el que un deudor a quien se permita pagar con retraso no se le debe permitir que no abone intereses. Desde ahora en adelante, no se podrá hacer así, siempre y cuando que el juicio de arbitraje haya comenzado antes de que efectúe su pago.

\section{Ayuda y supervisión por parte de la Corte}

La Corte Comercial es una sección especializada de la Corte inglesa y está a cargo de jueces con considerable experiencia en cuestiones jurídicas comerciales. La inmensa mayoría de los casos de naturaleza comercial se juzgan en esta Corte. A ésta se refieren todos los asuntos relativos a arbitraje comercial. Ya hemos mencionado dos casos en que la Corte Comercial tiene potestad para ayudar a la realizacion del arbitraje: en el nombramiento de árbitros y en dar la orden de aportación de garantía para los costos. También puede ayudar de otras maneras. Por ejemplo:

a) Puede obligar a un testigo a que preste declaración, siempre que esté dentro de su jurisdicción, o producir documentos que estén en su poder, dentro del juicio de arbitraje. Esto se hace por medio de una citación (15).

b) Junto con los árbitros, tiene potestad para ordenar que se co-

(13) Ley de 1950, Art. 20a.

(14) Ley de 1950, Art. $19^{\circ}$ A (Introducido por la Ley de Administración de Justicia de 1982, Art, 15\%).

(15) Ley de 1950 , Art. $12^{\circ}$ (4) 
muniquen documentos (11). Normalmente, se solicitaría al tribunal una orden de comunicación sólo en el caso de que los árbitros hayan visto rehusada su propia solicitud al efecto. El tribunal no se muestra nada favorable a interferir en las decisiones de los árbitros $\mathrm{y}$, para que tenga éxito una solicitud, los argumentos aportados tienen que ser muy convincentes.

c) En ciertas circunstancias tiene la potestad de dar una orden para que los árbitros tengan los mismos poderes que el tribunal mismo si el caso se estuviese viendo en la Corte Suprema, más bien que en arbitraje (16). El propósito es el de impedir retrasos innecesarios en el proceso de arbitraje, y la potestad se puede ejercer cuando los árbitros ya han dado orden contra una de las partes que haya desobedecido, por ejemplo, si los árbitros han dado una orden perentoria contra el demandante para que éste haga entrega de una reclamación y el demandante no lo haya hecho, el demandado (o los árbitros) podrá solicitar a la Corte que dé una orden otorgando derecho a los árbitros a desestimar la reclamación por carencia de acusación; o bien, en caso de que se haya desobedecido una orden de a portación de defensa, podrá tomar una decisión en favor del demandante).

El propósito fundamental más importante en que se basan los poderes de previsión de la Corte es asegurarse de que el arbitraje se realiza debidamente en interés de la justicia. Por ejemplo:

a) La potestad de retirar a un árbitro por proceder indebidamente (17), que se ejercerá en el momento en que haya mostrado una parcialidad práctica o posible o haya motivos serios para creer que o bien no puede o no podrá llevar adelante el procedimiento de forma equitativa y competente.

b) La Corte tiene la potestad de retirar a un árbitro cuando el ar-

(i6) Ley de 1979, Art. $5^{\circ}$ (1) y (2).

(17) Ley de 1950, Art. $23^{\circ}$ (1). 
bitraje, no progrese de forma expeditiva, dentro de lo razonable (18).

c) La potestad de terminar un juicio de arbitraje, ocupándose el mismo tribunal del asunto cuando haya habido alegato de fraude (19). Esta potestad sólo se puede ejercer cuando hay una solicitud procedente de la parte acusada de fraude (20). La razón es que la acusación de fraude es materia tan seria que una persona acusada de el deba tener derecho a que se dirima en un tribunal en vez de mediante el procedimiento particular que es el arbitraje.

(1) La potestad de anular una decisión arbitral, cuando el árbitro haya sido culpable de conducta incorrecta o la decisión se haya tomado de forma indebida (21).

e) La potestad de ordenar al árbitro que vuelva a estudiar una decisión que haya tomado (22). Por ciemplo, cuando un árbitro haya cometido un sencillo error y lo admita, el tribunal podrá ordenar que se le devuelva su decisión para permitirle corregir dicha falta, o bien se podrá ordenar la remisión en caso de que se hayan presentado diversos puntos contenciosos ante el árbitro y éste no haya decidido cosa alguna acerca de uno de ellos.

\section{Apelación de decisiones arbitrales}

Ll princip.t cambio que ha introducido la Ley de Arbitraje de 1979 ha siclo el relativo a las circunstancias en que una parte insatisfecha pueda apelar una decisión dada sobre la base de un error jurídico por parte del árbitro. Antes de la promulgación de dicha

(18) Ley de 1950 . Art. $13^{\circ}(3)$.

(19) Ley de 1950 , Art. $24^{\circ}$ (2)

(20) Véase el caso de Camilla Cotton Oil Co. v. Granadex S.A. y Tracomin S.A. (1976) 2 Ld. Rep. 10.

(21) Ley de 1950 , Art. $23^{\circ}$ (2)

(22) Ley de 1950 , Art. $22^{\circ}$ (1) 
ley, existía un procedimiento conocido como de "caso especial" o special case que permitía que las decisiones fuesen atacadas por causa de error. Existía la impresión de que se abusaba a menudo de este procedimiento, especialmente por parte de demandados perdedores de sus casos que deseaban posponer el momento de efectuar su pago al demandante. La Ley de 1979 abolió el procedimiento de caso especial y lo sustituyó por el de apelación limitada (23). No existe derecho de apelación. En su lugar existe la necesidad de obtener un permiso de la Corte misma antes de que se curse apelación alguna. Se ha producido cierto número de decisiones de las Cortes en relación a las circunstancias en que se concedió permiso de apelación. El resultado es que, a excepción de los casos en que está muy claro que la decisión de los árbitros no es correcta, es muy dificil obtener autorización para apelar, aún en casos en que la cuestión legal de que se trate es de importancia comercial general (24). No puede obtenerse una apelación sobre cuestiones de hecho. Lal cuestión de que se trate tiene que ser juridica $y$ hate poler afectar sensiblemente los derechos de una de las partes. Lat actitud de los tribunales ante esto es que los litigantes que hayan optado por someterse a arbitraje tienen derecho a esperar que la decisión arbitral sea definitiva y que no es función de las Cortes el remplazar su propio juicio por el de los árbitros.

Incluso las limitadas posibilidades de apelación arriba mencionadas pueden excluirse mediante acuerdo entre las partes (25). Pero hay tres categorias de contrato en que la exclusión no es posible a menos que tal acuerdo se alcance ante's del comienzo del arbitraje o en el caso de que el mismo contrato no esté gobernado por la ley inglesa: en primer lugar la mayor parte de los contratos marítimos, comprendidos contratos de fletamento y de carga, en segundo lugar, contratos de seguros $y$, por último contratos de mercancías (26).

(23) Iey de 1979, Art. $1^{\circ}$ y $2^{\circ}$.

(24) Véase, en particular, el "NEMA" (1981) 2 Ll. Rep. 239 y el "ANTAIOS” (1984) 2 LI. Rep. 235.

(25) Con arreglo a la Ley de 1979 , Art. $3^{\circ}$.

(26) Ley de 1979, Art. $4^{\circ}$. 
Ya hemos hablado de garantías acerca de los costos. La situación relativa a la garantía sobre la reclamación misma es muy diferente. La legislación inglesa no tiene ninguna disposición general para el otorgamiento de una garantía. No existe nada semejante al Saisie Conservatoire de Derecho Civil, aunque en muchas de tales jurisdicciones puede ser posible obtener subsanamiento de la garantía, relativa a un arbitraje en Londres. Sin embargo en Inglaterra existe un remedio o medida especial, que se conoce popularmente como "Mareva Injuction" (Requerimiento Judicial Mareva), que se puede obtener de las Cortes en relación a reclamaciones en arbitraje. Se trata de un requerimiento judicial que impide al demandado el traficar con sus bienes para burlar al demandante, algo semejante a un embargo. Se han producido numerosos casos de esta clase y la ley que a ellos se refiere es complicada. Nos limitaremos a mencionar la existencia del subsanamiento porque a veces puede ser útil. Aparte del procedimiento Mareva, el remedio de garantía restante de que se puede disponer para arbitraje marítimo es el arresto de buques.

En relación al cumplimiento de las decisiones arbitrales, todos los remedios disponibles para obligar al cumplimiento de una decisión dada pueden usarse también, por ejemplo, embargo por valor de la suma debida al demandado y sobre sus propiedades inmobiliarias. Puede ser de más interés para ustedes, considerando que los bienes del deudor estén fuera de Inglaterra, el saber que este país es consignatario de la Convención de Ginebra sobre cumplimiento de decisiones arbitrales extranjeras y de la Convención de Nueva York de 1958 para el reconocimiento y cumplimiento de decisiones arbitrales extranjeras, tal como también lo es Colombia (27). Esto significa que las decisiones inglesas pueden hacerse cumplir en muy distintos países. Además, por medio de un proceso muy sencillo es posible convertir una decisión arbitral inglesa en un juicio de un tribunal inglés para obligar al cumplimiento en países que observan tal formalidad.

(27) Que se ha prescrito en Inglaterra mediante la Ley de 1975. 